\title{
Electrostatic recognition and induced fit in the $\kappa$-PVIIA toxin binding to Shaker potassium channel
}

Xiaoqin Huang, Feng Dong, and Huan-Xiang Zhou

Table S1. Residue-Residue Contact Pairs Occurring in the Last 7.2 ns of MD

Simulations

\begin{tabular}{|c|c|c|c|}
\hline \multirow{2}{*}{ К-PVIIA residue } & \multirow{2}{*}{ Shaker residue } & \multicolumn{2}{|c|}{ Fraction $^{a}$} \\
\hline & & $5.0-\AA$ cutoff & $3.5-\AA$ cutoff \\
\hline \multirow{3}{*}{$\operatorname{Arg} 2$} & Asp447(A) & 0.97 & 0.97 \\
\hline & Phe425(B) & 1.00 & 1.00 \\
\hline & Val451(B) & 0.60 & 0.60 \\
\hline Ile3 & Phe425(A) & 0.55 & \\
\hline \multirow{2}{*}{ Asn5 } & Phe425(A) & 0.65 & \\
\hline & Asp447(D) & 0.82 & \\
\hline \multirow{13}{*}{ Lys7 } & Tyr445(A) & 1.00 & 1.00 \\
\hline & Gly446(A) & 1.00 & \\
\hline & Asp447(A) & 0.68 & \\
\hline & Phe425(B) & 0.88 & \\
\hline & Gly444(B) & 0.78 & \\
\hline & Tyr445(B) & 1.00 & 1.00 \\
\hline & Gly446(B) & 1.00 & \\
\hline & Asp447(B) & 0.69 & \\
\hline & Gly444(C) & 0.93 & \\
\hline & Tyr445(C) & 1.00 & 1.00 \\
\hline & Gly446(C) & 1.00 & \\
\hline & Tyr445(D) & 1.00 & 1.00 \\
\hline & Gly446(D) & 1.00 & \\
\hline \multirow{3}{*}{ Cys8 } & Phe425(B) & 0.95 & \\
\hline & Gly446(B) & 0.79 & \\
\hline & Asp447(B) & 1.00 & 0.45 \\
\hline Phe9 & Phe425(B) & 1.00 & 0.38 \\
\hline
\end{tabular}




\begin{tabular}{|c|c|c|c|}
\hline & Lys427(B) & 0.63 & \\
\hline & Asp447(B) & 1.00 & 0.50 \\
\hline Gln10 & Asn423(C) & 0.98 & 0.83 \\
\hline His11 & Glu422(B) & 0.97 & \\
\hline \multirow{2}{*}{ Leu 12} & Ser424(B) & 0.80 & \\
\hline & Phe425(B) & 1.00 & \\
\hline Asp13 & Glu422(B) & 1.00 & \\
\hline Arg18 & Glu422(B) & 1.00 & 1.0 \\
\hline Asn21 & Phe425(C) & 0.57 & \\
\hline \multirow{3}{*}{$\operatorname{Arg} 22$} & Phe425(C) & 0.97 & \\
\hline & Asp447(C) & 0.99 & \\
\hline & Glu422(D) & 1.00 & 0.99 \\
\hline \multirow{6}{*}{ Phe23 } & Gly446(C) & 0.94 & \\
\hline & Asp447(C) & 1.00 & 0.72 \\
\hline & Glu422(D) & 0.98 & \\
\hline & Asn423(D) & 1.00 & 0.62 \\
\hline & Thr449(D) & 0.81 & \\
\hline & Val451(D) & 0.89 & \\
\hline \multirow{5}{*}{ Asn24 } & Asp447(B) & 0.99 & \\
\hline & Gly446(C) & 1.00 & 0.96 \\
\hline & Asp447(C) & 1.00 & 0.38 \\
\hline & Met448(C) & 0.98 & \\
\hline & Thr449(C) & 0.98 & 0.60 \\
\hline \multirow{3}{*}{ Lys25 } & Asn423(D) & 0.54 & \\
\hline & Gly446(D) & 0.95 & 0.64 \\
\hline & Asp447(D) & 0.91 & \\
\hline
\end{tabular}

\footnotetext{
${ }^{a}$ Fraction of time a residue-residue contact was formed during the last $7.2 \mathrm{~ns}$ of the MD trajectory. Only contacts that were formed $>50 \%$ of time are listed.
} 
Table S2. Experimental and Calculated Effects (in kcal/mol) of K-PVIIA and Shaker Mutations on the Binding Affinity

\begin{tabular}{|c|c|c|}
\hline Mutation & $k_{\mathrm{B}} T \ln \left[\mathrm{IC}_{50}(\right.$ mutant $\left.) / \mathrm{IC}_{50}(\mathrm{wt})\right]$ & $\Delta \Delta G_{\mathrm{el}}{ }^{a}$ \\
\hline & к-PVIIA & \\
\hline $\operatorname{Arg} 2 \rightarrow$ Ala & $>4.01$ & $4.18 \pm 1.01$ \\
\hline Lys $7 \rightarrow$ Ala & $>4.01$ & $5.73 \pm 0.75$ \\
\hline Gln $10 \rightarrow$ Ala & 0.71 & $0.14 \pm 0.26$ \\
\hline Arg $18 \rightarrow$ Ala & 0.74 & $4.01 \pm 0.55$ \\
\hline Lys19 $\rightarrow$ Ala & 0.11 & $0.49 \pm 0.29$ \\
\hline $\operatorname{Arg} 22 \rightarrow$ Ala & 0.93 & $3.59 \pm 0.75$ \\
\hline Asn24 $\rightarrow$ Ala & 1.73 & $0.42 \pm 0.32$ \\
\hline \multirow[t]{2}{*}{ Lys $25 \rightarrow$ Ala } & 2.82 & $3.15 \pm 0.50$ \\
\hline & Shaker $\mathrm{K}^{+}$channel & \\
\hline Ser421 $\rightarrow$ Lys & 1.23 & $1.01 \pm 0.30$ \\
\hline Glu422 $\rightarrow$ Lys & 0.35 & $8.11 \pm 2.17$ \\
\hline Asn $423 \rightarrow$ Lys & 0.52 & $3.30 \pm 1.20$ \\
\hline Ser424 $\rightarrow$ Lys & 1.41 & $2.16 \pm 0.72$ \\
\hline Lys427 $\rightarrow$ Asp & -0.39 & $-2.54 \pm 0.44$ \\
\hline Lys427 $\rightarrow$ Asn & -0.50 & $-1.79 \pm 0.41$ \\
\hline Asp431 $\rightarrow$ Glu & 0.24 & $0.24 \pm 0.37$ \\
\hline Asp431 $\rightarrow$ Lys & 2.33 & $7.20 \pm 0.87$ \\
\hline Asp431 $\rightarrow$ Asn & 0.96 & $2.71 \pm 0.31$ \\
\hline Asp431 $\rightarrow$ Gln & 0.96 & $2.77 \pm 0.33$ \\
\hline Met448 $\rightarrow$ Lys & 0.24 & $3.68 \pm 0.52$ \\
\hline Thr449 $\rightarrow$ Ser & 1.06 & $0.21 \pm 0.34$ \\
\hline Gly452 $\rightarrow$ Glu & 0.15 & $-0.93 \pm 0.24$ \\
\hline Gly452 $\rightarrow$ Lys & 0.24 & $0.41 \pm 0.36$ \\
\hline Phe453 $\rightarrow$ Lys & 0.38 & $0.65 \pm 0.23$ \\
\hline Trp454 $\rightarrow$ Lys & 0.20 & $0.45 \pm 0.20$ \\
\hline
\end{tabular}

\footnotetext{
${ }^{a}$ Average and standard deviation calculated on 21 conformations are reported.
} 